Session 12-18

\title{
Silicon Wafer's Sample Thermal Diffusivity Determination Using Nano Flash Equipment
}

\author{
Kevin Bates, Samuel Ibekwe, and Guoqiang Li \\ Mechanical Engineering Department \\ Southern University, Baton Rouge \\ Kun Lian \\ Center for Advanced Microstructure Devices \\ Louisiana State University, Baton Rouge
}

\begin{abstract}
Silicon wafer was prepared for thermal diffusivity determination by Nano Flash Equipment. This involved clean room training, designing of masks using Auto Cad, repairing non-functional equipment prior to carrying out the experiment. This challenging and rewarding experience exposed me to what conducting a real research involves. It also gave me the confidence that I can make it in graduate education.
\end{abstract}

\section{Introduction}

Nanoflash LFA 447® is a flash diffusivity instrument which makes thermal properties testing fast, easy and affordable. Thermal diffusivity, conductivity, etc could be easily determined using this equipment. It consists of a Xenon flash lamp used to heat and read sample surfaces, which eliminates thermal resistance and thereby produces accurate measurement of thin samples $\sim 3 \mathrm{~mm}$. It has a broadband visible and near infrared wavelength with a variable pulse width of $100 \mu$ s, $400 \mu \mathrm{s}$ and $700 \mu$ s.

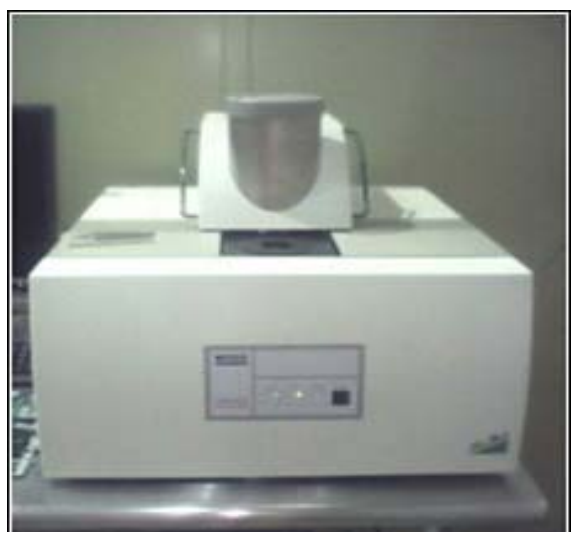

Figure 1. NETZSCH LFA 447® Nanoflash diffusivity instrument 


\section{Sample Design and Mask fabrication}

Specimen preparation as shown in Figure 2 involved microfabrication using LIGA techniques in a class 100 clean room. However, before the samples could be fabricated, a mask was made, which is like a negative for the optical imprints of the micro-sized specimens.

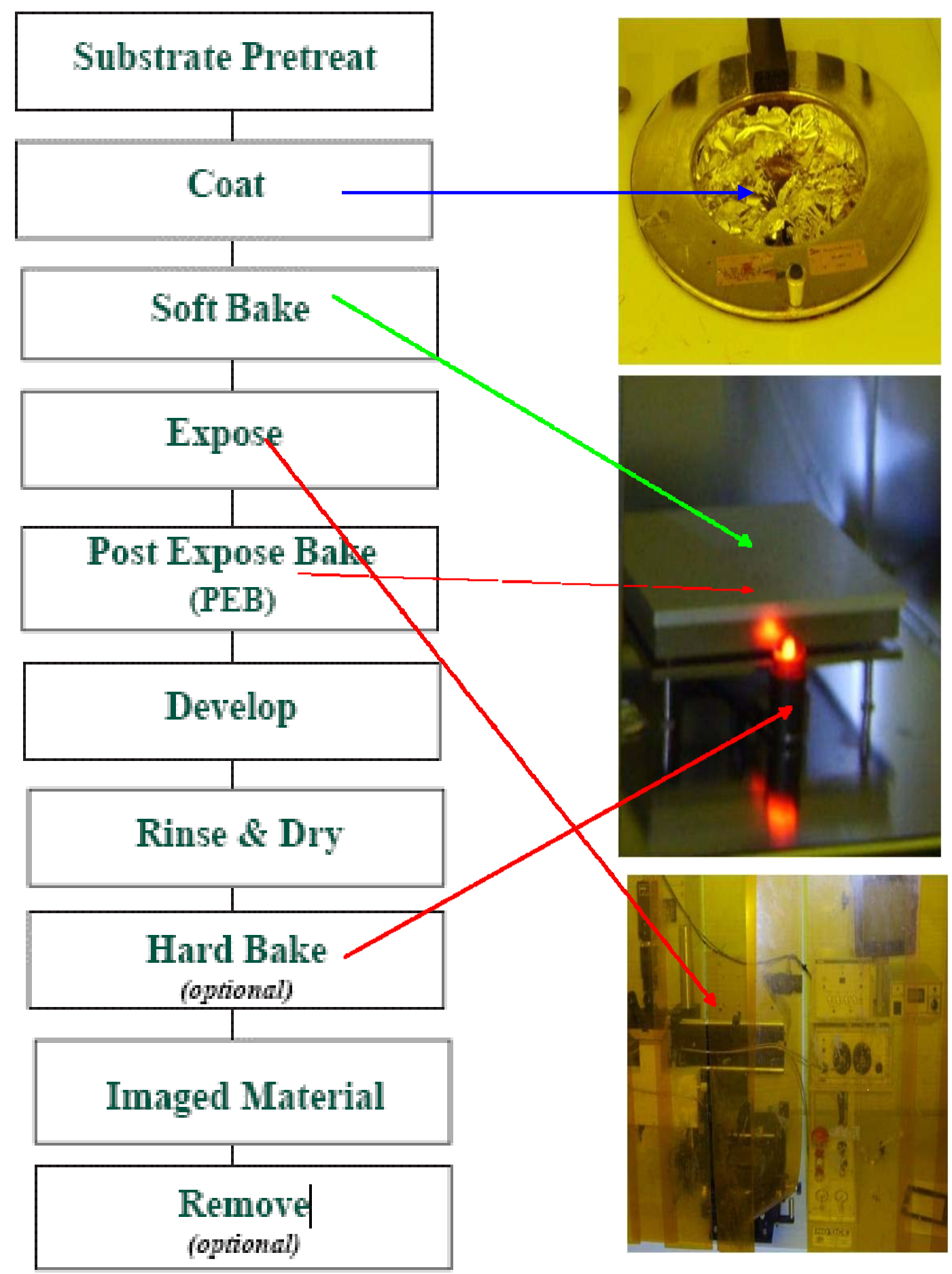

Figure 2. Schematic procedure for sample preparation 
Designing the mask started first with a two-hour "clean room" safety training. We were taught procedures for handling specimens safely, and how to conduct ourselves in the laboratory. Use of the equipment was clearly explained to us also by the CAMD personnel. Passing the radiation and clean room test certified me to use the laboratory. The $59.5 \mathrm{~mm}$ by $59.5 \mathrm{~mm}$ mask was first designed with AutoCAD ${ }^{\circledR}$ as shown in Figure 3 below. The file was then converted to a binary form to enable the pattern generator read and utilize it. GCA Mann $3600 \AA$ pattern generator was used to make the mask on a square $\mathrm{Cr} / \mathrm{Au}$ plate.
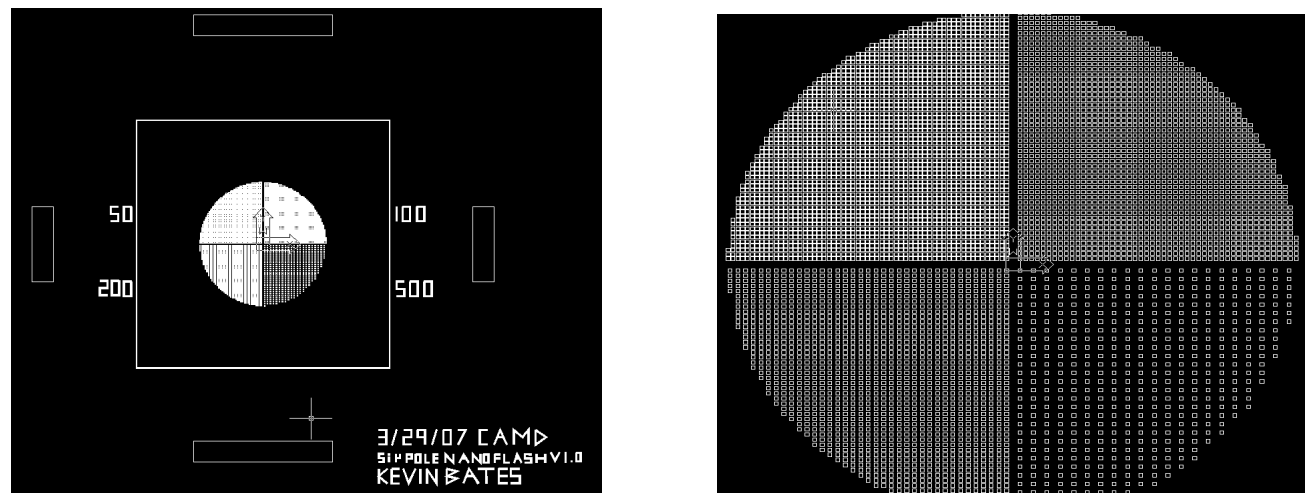

Figure 3. Mask design prior to optical printing of test samples.

The translucent samples were coated with gold layers on both sides. Thereafter a second layer of graphite about 5 microns thick was superimposed on the gold surface. This enhanced the absorption of flash energy, as well as the emission of infrared radiation waves.

\section{Summary and Conclusions}

While the sample preparation and tests are on-going, the results will be presented later. It has been a great educational experience for me. This project afforded me the opportunity to work with graduate students and professors in a research setting. My knowledge of computer-aided drafting came in handy, and it was gratifying to see an application of what I learned in a real world setting. Further, the interaction with other team members helped me learn valuable research procedures. Being trained and entrusted with complex laboratory equipment boosted my confidence and aspiration to go for my graduate program.

\section{References}

1. Puers B and Sansen W, Compensation structures for convex corner micromachining in silicon, Sensors and Actuators, A21 - A23, 1990, p1036 - 1041.

2. Shur, M., Physics of Semiconductor Devices, Prentice Hall, 1990.

3. Jerias Batista, Temperature dependence of carrier mobility in Si wafer measured by infrared photocarrier radiometry, Applied Physics Letters, 2003. 


\section{Acknowledgement}

This study was sponsored by a grant from U.S. Department of Energy, Award No. DE-FG5205N27041. The authors also acknowledge support of Mechanical Engineering Department, Southern University, Baton Rouge, and CAMD, Louisiana State University, Baton Rouge. The author is grateful to Mr. Shannun T. Walker for his assistance.

\section{KEVIN BATES}

Mr. Bates is a senior student at Southern University, Baton Rouge, Louisiana.

SAMUEL IBEKWE, GUOQIANG LI, AND KUN LIAN

Drs. Samuel Ibekwe and Guoqiang Li are professors of Mechanical Engineering at Southern University, Baton Rouge, while Dr. Kun Lian is a professor at Louisiana State University, Baton Rouge, LA, and is affiliated with Center for Advance Micro Devices (CAMD) in Baton Rouge. 